\title{
Efeitos da metadona ou do neostigmine, associados à lidocaína administrados pela via epidural em cães
}

\author{
[Effects of methadone or neostigmine in combination with lidocaine \\ for epidural anesthesia in dogs] \\ E.R. Monteiro ${ }^{1}$, R.J.O. Dossi ${ }^{1}$, A.P. Antunes ${ }^{1}$, D. Campagnol $^{2}$, \\ C.M. Bettini ${ }^{1}$, J.C. Choma ${ }^{1}$ \\ ${ }^{1}$ Centro Universitário de Maringá - CESUMAR \\ Av. Guedner 1610 - Zona 8 \\ 87050-900 - Maringá, PR \\ ${ }^{2}$ Faculdade de Medicina Veterinária e Zootecnia - UNESP - Botucatu, SP
}

\begin{abstract}
RESUMO
Seis cães adultos, de raças e sexos variados, com peso de $13,3 \pm 3,4 \mathrm{~kg}$ (média $\pm \mathrm{DP}$ ), foram utilizados no estudo. Os animais foram tranqüilizados com acepromazina $(0,1 \mathrm{mg} / \mathrm{kg}$, IV) e, após 30 minutos, foram aleatoriamente submetidos à anestesia epidural com um dos seguintes tratamentos: lidocaína $2 \%$ $0,25 \mathrm{ml} / \mathrm{kg}$ (controle); neostigmine $0,01 \mathrm{mg} / \mathrm{kg}$ +lidocaína (NEO); metadona $0,3 \mathrm{mg} / \mathrm{kg}+$ lidocaína (MET). Todos os animais foram submetidos aos três tratamentos com intervalo mínimo de uma semana. Foram mensuradas as freqüências cardíaca (FC) e respiratória (FR), a pressão arterial sistólica (PAS), o tempo para a perda do reflexo interdigital, a duração e a altura do bloqueio sensitivo, durante um período de 90 minutos. Não houve diferença significativa entre os tratamentos nos valores de FC, PAS e FR, bem como na duração do bloqueio sensitivo e no tempo para a perda do reflexo interdigital. No grupo MET, houve diminuição de $\mathrm{FC}$ dos 30 aos 90 minutos em relação ao valor basal. Bloqueio sensitivo mais cranial também foi observado em MET. A associação de neostigmine ou metadona não prolongou o período hábil de anestesia epidural produzido pela lidocaína em cães. A metadona, mas não o neostigmine, parece estender mais cranialmente o bloqueio epidural pela lidocaína.
\end{abstract}

Palavras-chave: cão, anestesia epidural, metadona, neostigmine, lidocaína

\begin{abstract}
Six mature mongrel dogs of both genders, weighing 13.3 $\pm 3.4 \mathrm{~kg}$ (mean $\pm S D$ ) were used in the present research. Thirty minutes after premedication with intravenous acepromazine $(0.1 \mathrm{mg} / \mathrm{kg}, I \mathrm{~V})$, dogs were randomly assigned to receive epidural administration of one of following three treatments: $2 \%$ lidocaine $0.25 \mathrm{ml} / \mathrm{kg}$ (control), or neostigmine $0.01 \mathrm{mg} / \mathrm{kg}$ plus lidocaine (NEO), or methadone $0.3 \mathrm{mg} / \mathrm{kg}$ plus lidocaine (MET). All dogs received all treatments in a cross-over design with at least one-week interval. Heart rate (HR), respiratory rate (RR), systolic arterial pressure (SAP), time to loss of pedal withdrawal reflex, duration of epidural anesthesia, and cranial spread of epidural anesthesia were evaluated for 90 minutes. No differences among treatments in HR, RR, SAP, duration of anesthesia, and time to loss of pedal withdrawal reflex were found. In MET, HR decreased from 30 to 90 minutes compared to baseline and there was a higher cranial spread of epidural anesthesia than in controls and NEO animals. Neostigmine or methadone did not prolong epidural anesthesia with lidocaine in dogs. Methadone, but not neostigmine, appeared to result in more cranial spread of epidural anesthesia with lidocaine.
\end{abstract}

Keywords: dog, epidural anesthesia, methadone, neostigmine, lidocaine

Recebido em 15 de janeiro de 2007

Aceito em 12 de outubro de 2008

E-mail: raposo@cesumar.br 


\section{INTRODUÇÃO}

A anestesia epidural é uma técnica de anestesia regional, que consiste na deposição de anestésico local ao redor da dura-máter, resultando em difusão longitudinal do anestésico no interior do espaço epidural e bloqueio das raízes sensitivas e motoras dos nervos espinhais (Massone, 2003).

Em cães, a medula espinhal, bem como o espaço subaracnóideo, termina no nível da sétima vértebra lombar (L7) (Grimm, 2004). Esse fato torna a anestesia epidural uma técnica segura e eficaz para a realização de procedimentos na região da cauda, períneo, membros pélvicos e intervenções no abdome baixo de cães (Massone, 2003).

A anestesia epidural pode ser utilizada como técnica anestésica isolada para a realização de procedimentos cirúrgicos, sendo, neste caso, recomendada a sedação do animal para minimizar o desconforto durante a introdução da agulha e injeção da solução anestésica no espaço epidural (Massone, 2003). Assim como outras técnicas de anestesia local, também pode ser utilizada como adjuvante da anestesia injetável ou inalatória para melhorar a analgesia transoperatória e diminuir o requerimento de anestésicos injetáveis e voláteis.

Entre os anestésicos locais utilizados para anestesia epidural, destacam-se a lidocaína e a bupivacaína. A anestesia epidural com lidocaína na concentração de $2 \%$, sem a associação de vasoconstritor, produz um período hábil que varia de 45 a 120 minutos (Massone, 2003).

O uso de opióides pela via epidural também vem sendo extensivamente estudado no cão. A administração epidural de opióides resulta em potencialização da analgesia e menor consumo de analgésicos no período pós-operatório (Troncy et al., 2002; Valadão et al., 2002; Fowler et al., 2003). Analgesia prolongada, por até 24 horas, foi atribuída à administração epidural de morfina (Valadão et al., 2002; Fowler et al., 2003). Além do efeito analgésico prolongado, a associação de opióides a anestésicos locais pela via epidural visa aumentar a duração do bloqueio sensitivo e estendê-lo a segmentos espinhais mais craniais (Wetmore e Glowaski, 2000). Estudos em cães comprovaram que o butorfanol e a buprenorfina prolongaram o período hábil proporcionado pela lidocaína, enquanto somente o butorfanol estendeu cranialmente a altura do seu bloqueio (Ishiy et al., 2002; Campagnol et al., 2004). As doses de opióides utilizadas por essa via são geralmente baixas, podendo corresponder a $1 / 10$ da dose utilizada pelas vias parenterais, dependendo do fármaco utilizado. Dessa forma, a incidência de efeitos adversos resultantes da administração de opióides pela via epidural é baixa. No entanto, retenção urinária, prurido e vômito foram relatados após a administração epidural de opióides (Gedney e Liu, 1998).

A metadona é um opióide que apresenta efeitos farmacológicos similares aos da morfina. Esse fármaco atua em receptores opióides do tipo $\mu$, sendo esse o provável mecanismo pelo qual provoca seus efeitos analgésicos (Gutstein e Akil, 2001). Acredita-se, ainda, que a metadona possa atuar como antagonista de receptores Nmetil-D-aspartato (NMDA) na medula espinhal, característica essa que pode incrementar a analgesia proporcionada por esse fármaco e diminuir a ocorrência de tolerância pelo seu uso contínuo (Wagner, 2002).

No homem, a metadona demonstrou proporcionar analgesia similar à morfina, à meperidina e ao fentanil quando administrados por via epidural (Torda e Pybus, 1982). A analgesia pós-operatória após administração da metadona por essa via foi considerada satisfatória após diversos procedimentos cirúrgicos, tais como toracotomia (Parramon et al., 2003), laparotomia (Prieto-Alvarez et al., 1997) e cirurgia corretiva de hérnia de disco lombar (Hernandez-Palazon et al., 1997). A incidência de efeitos adversos após a administração epidural de metadona foi baixa e consistiu em depressão respiratória (PrietoAlvarez et al., 1997; Shir et al., 2001), náuseas e prurido (Haynes et al., 1993), sendo a incidência de alguns desses efeitos mais baixa quando comparada à de outros opióides (morfina e fentanil) pela mesma via (Gedney e Liu, 1998).

Em eqüinos, a administração de metadona por via epidural, na dose de $0,1 \mathrm{mg} / \mathrm{kg}$, proporcionou analgesia no períneo, região lombossacra e torácica, com duração de 5 horas e ausência de efeitos adversos (Olbrich e Mosing, 2003). Em gatos, a metadona administrada por via epidural proporcionou analgesia de duração similar à 
meperidina (Tung e Yaksh, 1982). No entanto, há carência de estudos sobre o uso da metadona pela via epidural na espécie canina.

O neostigmine é um agente da classe dos anticolinesterásicos. Esses fármacos são utilizados clinicamente no tratamento do íleo paralítico, atonia vesical, glaucoma e miastenia gravis, bem como na reversão do bloqueio neuromuscular proporcionado pela administração de agentes curarizantes durante a anestesia. Os fármacos dessa classe atuam ao inibir reversivelmente a ação da acetilcolinesterase, causando acúmulo de acetilcolina em gânglios autonômicos, receptores muscarínicos do sistema nervoso autônomo, receptores nicotínicos na placa motora e receptores colinérgicos no sistema nervoso central (Taylor, 2001).

Estudos no homem têm demonstrado que a administração de neostigmine por via epidural proporciona analgesia (Lauretti et al., 1999; Kaya et al., 2004; Masaki et al., 2004). Adicionalmente, a associação de neostigmine a anestésicos locais por essa via levou ao aumento da duração da analgesia produzida por esses fármacos e à diminuição do consumo total de analgésicos durante as primeiras 24 horas após intervenções cirúrgicas (Lauretti et al., 1999; Nakayama et al., 2001). Sedação (Kaya et al., 2004), náuseas (D'Angelo et al., 2001; Omais et al., 2002) e vômito (Omais et al., 2002) foram os efeitos adversos atribuídos à administração epidural de neostigmine no homem.

Em ovelhas, a administração de neostigmine por via intratecal promoveu analgesia especialmente no período pós-operatório imediato (até 48 horas). Tais resultados não foram confirmados após administração de doses similares no quinto dia após o procedimento cirúrgico. O mesmo estudo evidenciou ainda que a analgesia proporcionada pelo neostigmine se deve provavelmente à ação em receptores muscarínicos do tipo M1 (Bouaziz et al., 1995). Assim como acontece com a metadona, há carência de estudos sobre a administração epidural de neostigmine em cães.
O presente estudo teve como objetivo estudar os efeitos da associação de neostigmine ou metadona à lidocaína na anestesia epidural lombossacra em cães.

\section{MATERIAL E MÉTODOS}

O estudo foi aprovado pelo Comitê de Ética em Experimentação Animal da Universidade Estadual de Londrina (protocolo n ${ }^{\circ} 29 / 2005$ ). Foram utilizados seis cães (cinco fêmeas e um macho) sem raça definida, adultos, porém com idades variadas e peso de $13,3 \pm 3,4 \mathrm{~kg}$ (média \pm DP). A seleção dos animais foi baseada em exame clínico e hemograma completo com valores dentro dos limites considerados fisiológicos. Foram descartados do estudo animais obesos, com deformidades na pelve ou na coluna vertebral. Os animais foram submetidos a jejum alimentar de 12 horas, não sendo, no entanto, privados de água.

No dia do experimento, os animais foram pesados e tranqüilizados com acepromazina ${ }^{1}$ na dose de $0,1 \mathrm{mg} / \mathrm{kg}$ pela via intravenosa. Decorridos 30 minutos, os animais foram posicionados em decúbito esternal e, após tricotomia e anti-sepsia na região lombossacra, cada animal recebeu, aleatoriamente, pela via epidural, um dos seguintes tratamentos: lidocaína $2 \%$ sem vasoconstritor ${ }^{2} \quad 0,25 \mathrm{ml} / \mathrm{kg}$ (grupocontrole); neostigmine ${ }^{3} 0,01 \mathrm{mg} / \mathrm{kg} \quad(0,02 \mathrm{ml} / \mathrm{kg})$ associado à lidocaína (grupo NEO); metadona ${ }^{4}$ $0,3 \mathrm{mg} / \mathrm{kg} \quad(0,03 \mathrm{ml} / \mathrm{kg})$ associada à lidocaína (grupo MET). Nos animais dos grupos NEO e MET, o volume de lidocaína utilizado foi o suficiente para se obter um volume final equivalente a $0,25 \mathrm{ml} / \mathrm{kg}$ de solução. Para a punção do espaço epidural, lombossacro, foi utilizada agulha hipodérmica de calibre 40x8. O correto posicionamento da agulha no espaço epidural foi confirmado: 1) pela penetração da agulha através do ligamento amarelo, percebida como uma sensação de crepitação ao perfurar esse ligamento; 2) pela aspiração de uma gota de solução salina $(\mathrm{NaCl} \quad 0,9 \%)$, depositada no

\footnotetext{
${ }^{1}$ Acepran 0,2\%, Univet/Vetnil - São Paulo, Brasil.

${ }^{2}$ Xylestesin 2\% sem vasoconstritor, Cristália Produtos

Químicos Farmacêuticos Ltda. - Itapira, Brasil.

${ }^{3}$ Prostigmine solução injetável $0,5 \mathrm{mg} / \mathrm{ml}$, ICN Farmacêutica

Ltda. - Campinas, Brasil.

${ }^{4}$ Metadon solução injetável $10 \mathrm{mg} / \mathrm{ml}$, Cristália Produtos

Químicos Farmacêuticos Ltda. - Itapira, Brasil.
} 
canhão da agulha, para o interior do espaço epidural devido à existência de pressão negativa nesse espaço; e 3) pela ausência de resistência no momento da injeção. Após a injeção epidural, os animais foram mantidos em decúbito esternal por 15 minutos, após o que foram posicionados em decúbito lateral direito até o final do estudo. Todos os animais foram submetidos aos três tratamentos, com intervalo mínimo de uma semana entre cada experimento. Foram avaliadas as freqüências cardíaca (FC) e respiratória (FR), a pressão arterial sistólica (PAS), a altura do bloqueio e a duração do bloqueio sensitivo. A freqüência cardíaca foi mensurada por auscultação e a FR, por visualização. A mensuração da PAS foi feita pelo método indireto, com auxílio de um doppler ${ }^{5}$ vascular. A altura de bloqueio foi avaliada pelo pinçamento da pele na região do dorso (sobre a linha média), com auxílio de uma pinça hemostática (fechada até a terceira cremalheira). Contrações da musculatura dorsal ou alterações comportamentais em resposta ao pinçamento foram consideradas respostas positivas. A duração do bloqueio foi baseada na avaliação do reflexo interdigital nos membros pélvicos. Esse reflexo foi avaliado por meio do pinçamento da prega interdigital com um pinça hemostática (até a terceira cremalheira) e considerado presente quando da retirada do membro ou em caso de manifestações de desconforto do animal. A duração do bloqueio foi definida como o tempo decorrido entre a perda do reflexo interdigital até a recuperação desse reflexo. Para determinar o momento de recuperação do reflexo interdigital em cada membro, utilizou-se a média aritmética entre o primeiro momento em que houve reflexo presente e o momento anterior (em que o reflexo esteve ausente). Por exemplo, se o reflexo foi presente pela primeira vez aos 90 minutos de anestesia, considerou-se que a recuperação desse reflexo ocorreu aos 83 minutos (média entre o momento em que o reflexo esteve presente [90 minutos] e o momento anterior no qual o reflexo esteve ausente [75 minutos]). Caso a duração do bloqueio tenha sido diferente para os membros pélvicos direito e esquerdo, considerou-se a duração do bloqueio epidural como sendo a média aritmética entre as durações dos bloqueios em cada membro.

O estudo foi do tipo cego, de forma que o avaliador não sabia a qual dos tratamentos o animal foi submetido. As variáveis FC, PAS e FR foram mensuradas imediatamente antes da realização da anestesia epidural (basal) e após $15,30,45,60,75$ e 90 minutos. A altura de bloqueio foi mensurada nos mesmos momentos mencionados e adicionalmente a cada 15 minutos até a recuperação do reflexo interdigital em ambos os membros pélvicos.

A análise estatística foi realizada com auxílio de um programa estatístico (Graphpad Prism 4). Diferenças entre grupos para as variáveis $\mathrm{FC}$, PAS e FR foram realizadas por meio da análise de variância em dois critérios de classificação, seguida pela correção de Bonferroni. Diferenças dentro de cada grupo em relação aos valores basais foram realizadas por meio da análise de variância para amostras repetidas, seguida pelo teste Dunnett. As comparações entre grupos para a duração do bloqueio e tempo para perda do reflexo interdigital foram realizadas por meio da análise de variância para amostras repetidas. Os dados de altura de bloqueio não foram analisados estatisticamente. Todos os testes foram realizados a 5\% de significância.

\section{RESULTADOS}

Não houve diferença significativa entre os grupos para os valores de FC, PAS e FR (Tab. 1). No entanto, houve diminuição de FC em relação aos valores basais no grupo MET de 30 a 90 minutos após a administração epidural. A duração do bloqueio sensitivo bem como o tempo para a perda do reflexo interdigital não diferiram entre os grupos (Tab. 2; Fig. 1). Embora os dados obtidos na mensuração da altura de bloqueio não tenham sido analisados estatisticamente, foi possível observar um bloqueio sensitivo mais cranial nos animais do grupo MET em relação aos grupos controle e NEO (Tab. 3).

\footnotetext{
${ }^{5}$ Doppler model 841-A, Parks Medical Electronics - Aloha, EUA.
} 
Tabela 1. Freqüência cardíaca (FC), pressão arterial sistólica (PAS) e freqüência respiratória (FR) (média \pm desvio-padrão) em cães tranqüilizados com acepromazina antes (basal) e submetidos à anestesia epidural com lidocaína $2 \%$ sem vasoconstritor (controle), com neostigmine associado à lidocaína (NEO) ou com metadona associada à lidocaína (MET)

\begin{tabular}{lccccccc}
\hline & Basal & 15 & 30 & 45 & 60 & 75 & 90 \\
\hline Controle & $87 \pm 27$ & $86 \pm 21$ & $85 \pm 22$ & $86 \pm 20$ & $89 \pm 17$ & $92 \pm 26$ & $95 \pm 28$ \\
NEO & $91 \pm 15$ & $77 \pm 12$ & $85 \pm 13$ & $86 \pm 7$ & $85 \pm 9$ & $81 \pm 9$ & $89 \pm 20$ \\
MET & $105 \pm 17$ & $85 \pm 28$ & $77 \pm 15^{*}$ & $83 \pm 23^{*}$ & $79 \pm 19^{*}$ & $76 \pm 11^{*}$ & $77 \pm 14^{*}$ \\
\hline \multicolumn{7}{c}{ PAS (mmHg) } \\
\hline Controle & $117 \pm 24$ & $112 \pm 17$ & $111 \pm 15$ & $115 \pm 11$ & $113 \pm 14$ & $112 \pm 12$ & $109 \pm 13$ \\
NEO & $108 \pm 12$ & $108 \pm 7$ & $109 \pm 16$ & $108 \pm 17$ & $113 \pm 20$ & $110 \pm 15$ & $112 \pm 13$ \\
MET & $107 \pm 9$ & $113 \pm 16$ & $109 \pm 13$ & $109 \pm 14$ & $107 \pm 13$ & $108 \pm 9$ & $110 \pm 12$ \\
\hline \multicolumn{7}{c}{ FC (bpm) } \\
\hline Controle & $22 \pm 7$ & $18 \pm 7$ & $18+5$ & $18 \pm 4$ & $18 \pm 6$ & $22 \pm 9$ & $25 \pm 14$ \\
NEO & $21 \pm 5$ & $19 \pm 7$ & $20 \pm 10$ & $19 \pm 7$ & $19 \pm 10$ & $18 \pm 6$ & $19 \pm 9$ \\
MET & $29 \pm 22$ & $21 \pm 16$ & $25 \pm 19$ & $23 \pm 18$ & $25 \pm 16$ & $21 \pm 11$ & $22 \pm 17$ \\
\hline
\end{tabular}

*Diferença em relação ao valor basal (teste Dunnett, $\mathrm{P}<0,05)$.

Tabela 2. Período de latência do reflexo interdigital e duração do bloqueio epidural (média \pm desviopadrão) em cães tranqüilizados com acepromazina e submetidos à anestesia epidural com lidocaína $2 \%$ sem vasoconstritor (controle), com neostigmine associado à lidocaína (NEO) ou com metadona associada à lidocaína (MET)

\begin{tabular}{llll}
\hline & Controle & NEO & MET \\
\hline Período de latência do reflexo interdigital (min) & $7,5 \pm 1,9$ & $6,4 \pm 2,0$ & $6,0 \pm 1,8$ \\
& & & \\
Duração do bloqueio (min) & $88 \pm 24$ & $76 \pm 39$ & $77 \pm 29$ \\
\hline
\end{tabular}

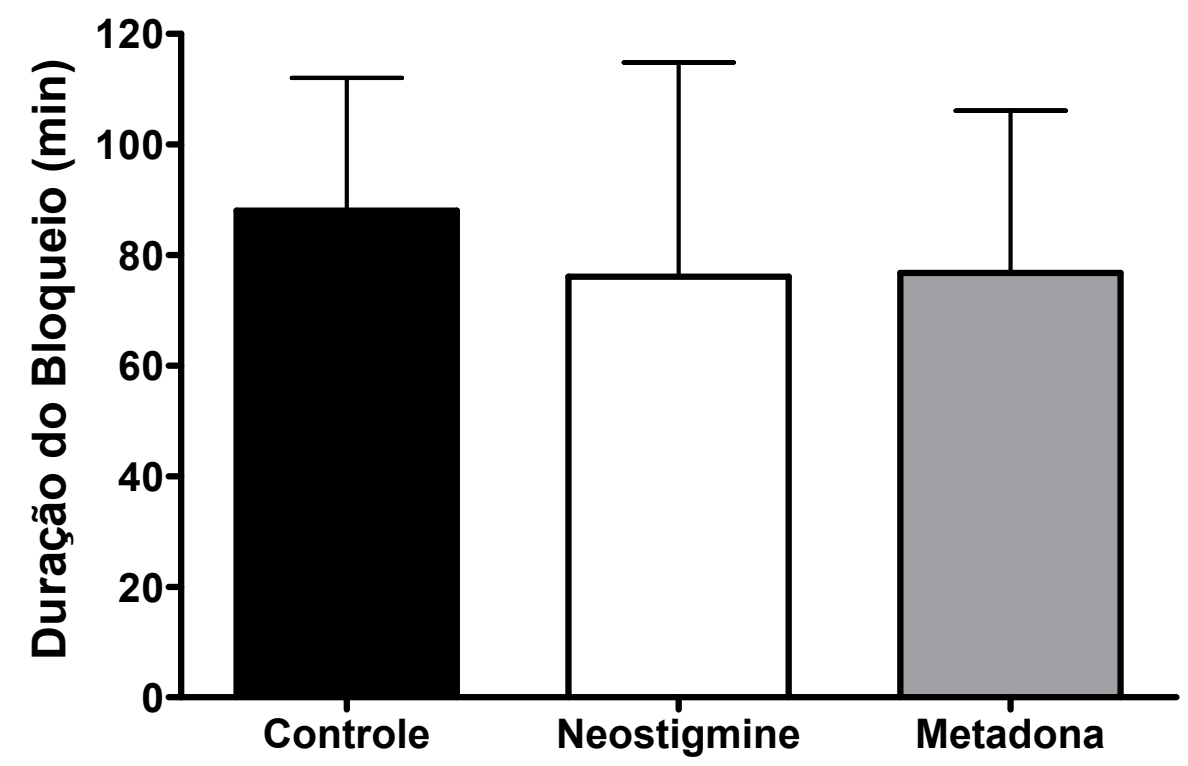

Figura 1. Duração do bloqueio epidural (média \pm desvio-padrão) em cães tranqüilizados com acepromazina e submetidos à anestesia epidural com lidocaína $2 \%$ sem vasoconstritor (controle), com neostigmine associado à lidocaína ou com metadona associada à lidocaína. 
Tabela 3. Altura máxima de bloqueio sensitivo em cães tranqüilizados com acepromazina e submetidos à anestesia epidural com lidocaína $2 \%$ sem vasoconstritor (controle), com neostigmine associado à lidocaína (NEO) ou com metadona associada à lidocaína (MET)

\begin{tabular}{cccc}
\hline Animal & Controle & NEO & MET \\
\hline 1 & L5 & L7 & L3 \\
2 & L7 & L4 & T12 \\
3 & L6 & L6 & L3 \\
4 & L3 & L6 & T10 \\
5 & L7 & L6 & L2 \\
6 & L4 & L5 & L5 \\
\hline
\end{tabular}

\section{DISCUSSÃO}

A deposição de anestésicos locais no espaço epidural pode resultar em bloqueio de fibras préganglionares simpáticas (Massone, 2003; Grimm, 2004). Esse efeito pode resultar em diminuição de até $20 \%$ na pressão arterial e não parece ser influenciado pela altura do bloqueio (Hall et al., 2001). A diminuição na pressão arterial, porém, é bem tolerada por animais hígidos (Hall et al., 2001). Esses resultados foram confirmados no presente estudo, isto é, não foi evidenciada diminuição da PAS após a administração dos tratamentos controle, NEO ou MET, mesmo que o bloqueio sensitivo tenha se estendido mais cranialmente nos animais do grupo MET em relação aos dos demais grupos.

Os resultados do presente estudo confirmam os relatos anteriores quanto à baixa incidência de efeitos adversos relacionados à administração epidural de metadona (Prieto-Alvarez et al., 1997; Gedney e Liu, 1998; Shir et al., 2001; Leibetseder et al., 2006) ou neostigmine (Lauretti et al., 1999; Omais et al., 2002). Embora náuseas e vômito tenham sido relatados após a administração epidural de neostigmine no homem (Owen et al., 2000; D'Angelo et al., 2001), esse efeito não foi observado em nenhum dos animais no presente estudo. Apesar de ter havido diminuição de FC nos animais do grupo MET, somente um animal apresentou bradicardia (FC $<60 \mathrm{bpm}$ ), cuja duração foi de 30 minutos. Esse fato não parece apresentar importância clínica em animais hígidos, uma vez que a pressão arterial sistólica se manteve sempre acima de valores considerados aceitáveis (PAS $\geq 90 \mathrm{mmHg}$ ). Diminuição na $\mathrm{FC}$ sem a ocorrência de bradicardia severa também foi observada por Leibetseder et al. (2006) em cães tratados com metadona $(0,3 \mathrm{mg} / \mathrm{kg})$ pela via epidural e anestesiados com isoflurano.

Quanto à duração do bloqueio ( $88 \pm 24$ minutos) e o tempo para a perda do reflexo interdigital (7,5 $\pm 1,9$ minutos) produzidos pela anestesia epidural com lidocaína, são similares os resultados ao relatados anteriormente (Massone, 2003; Campagnol et al., 2004).

A associação do butorfanol e da buprenorfina à lidocaína na anestesia epidural lombossacra em cães resultou em aumento na duração do bloqueio sensitivo (Campagnol et al., 2004). Adicionalmente, o butorfanol resultou em bloqueio mais cranial, a ponto de permitir a realização de ovariosalpingohisterectomia em cadelas (Ishiy et al., 2002; Campagnol et al., 2004). No entanto, quando o opióide associado foi o tramadol, não se evidenciou aumento no tempo de bloqueio sensitivo nem na altura do bloqueio (Campagnol et al., 2004). No presente estudo, a associação da metadona à lidocaína resultou em bloqueio sensitivo mais cranial em relação ao uso isolado da lidocaína. Porém, a duração do bloqueio não foi influenciada pela metadona. É possível que essas diferenças observadas entre os diversos opióides sobre a anestesia epidural pela lidocaína estejam relacionadas à afinidade de cada fármaco pelos diferentes receptores opióides. Outra possibilidade para explicar tais divergências seria a diferença nas características farmacocinéticas (lipo e hidrossolubilidade) de cada opióide. Estudos futuros são necessários para esclarecer os mecanismos pelos quais os opióides podem produzir aumento na duração e extensão do bloqueio epidural produzido pela lidocaína.

Estudos realizados em pacientes humanas submetidas a cirurgias abdominais demonstraram que o neostigmine, em dose similar à utilizada no presente estudo, prolongou a analgesia proporcionada pela administração epidural da bupivacaína (Kirdemir et al., 2000; Nakayama et al., 2001). Em outro estudo, realizado em pacientes humanos submetidos à cirurgia no joelho, o neostigmine prolongou o efeito analgésico da lidocaína de forma não dosedependente (Lauretti et al., 1999). Em cadelas submetidas à ovariosalpingohisterectomia, a administração epidural de neostigmine isolado ou associado à morfina resultou em menor consumo de analgésicos no período pós- 
operatório (Marucio et al., 2006). No presente estudo, o neostigmine não interferiu com a duração ou a altura do bloqueio epidural produzido pela lidocaína. Porém, não se pode afirmar, a partir desses resultados, que a administração epidural de neostigmine não tenha resultado em melhor analgesia do que a lidocaína isolada, uma vez que o estímulo utilizado evidencia apenas a presença ou a ausência de bloqueio sensitivo. A avaliação do reflexo interdigital não permite quantificar diferentes níveis de analgesia. Portanto, seriam necessários estudos futuros para avaliar se a associação do neostigmine à lidocaína resulta em melhor efeito analgésico do que a lidocaína isolada.

\section{CONCLUSÕES}

A associação de neostigmine $(0,01 \mathrm{mg} / \mathrm{kg})$ ou metadona $(0,3 \mathrm{mg} / \mathrm{kg})$, na anestesia epidural lombossacra em cães, não prolongou o tempo de bloqueio sensitivo produzido pela lidocaína. Enquanto o neostigmine não demonstrou interferir na altura de bloqueio, a associação da metadona à lidocaína pareceu resultar em bloqueio sensitivo mais cranial.

\section{REFERÊNCIAS BIBLIOGRÁFICAS}

BOUAZIZ, H.; TONG, C.; EISENACH, J.C. Postoperative analgesia from intrathecal neostigmine in sheep. Anesth. Analg., v.80, p.1140-1144, 1995.

CAMPAGNOL, D.; MONTEIRO, E.R.; LUNA, S.P.L. et al. Efeitos cardiorrespiratório e analgésico da clonidina, xilazina, butorfanol, buprenorfina e tramadol, associados à lidocaína, na anestesia epidural de cães. Braz. J. Vet. Res. Anim. Sci., v.41, p.41-42, 2004.

D'ANGELO, R.; DEAN, L.S.; MEISTER, G.C. et al. Neostigmine combined with bupivacaine, clonidine, and sufentanil for spinal labor analgesia. Anesth. Analg., v.93, p.1560-1564, 2001.

FOWLER, D.; ISAKOW, K.; CAULKETT, N. et al. An evaluation of the analgesic effects of meloxicam in addition to epidural morphine/mepivacaine in dogs undergoing cranial cruciate ligament repair. Can. Vet. J., v.44, p.643-648, 2003.
GEDNEY, J.A.; LIU, E.H. Side-effects of epidural infusions of opioid bupivacaine mixtures. Anaesthesia, v.53, p.1148-1155, 1998.

GRIMM, K.A. Anestesia epidural. In: GREENE, S.A. (Ed). Segredos em anestesia veterinária e manejo da dor. Porto Alegre: Artmed, 2004. p.387-392.

GUTSTEIN, H.B.; AKIL, H. Opioid analgesics. In: HARDMAN, J.G.; LIMBIRD, L.E. (Eds). Goodman \& Gilman's: the pharmacological basis of therapeutics. 10.ed. New York: McGraw-Hill, 2001. p.569-619.

HAYNES, S.R.; DAVIDSON, I.; ALLSOP, J.R. et al. Comparison of epidural methadone with epidural diamorphine for analgesia following caesarean section. Acta Anaesthesiol. Scand., v.37, p.375-380, 1993.

HALL, L.W.; CLARKE, K.W.; TRIM, C.M. General principles of local analgesia. In: HALL, L.W.; CLARKE, K.W.; TRIM, C.M. (Eds). Veterinary anaesthesia. 10.ed. London: W.B. Saunders, 2001. p.225-245.

HERNANDEZ-PALAZON, J.; TORTOSASERRANO, J.A.; SANCHEZ-ORTEGA, J.L. et al. Postoperative analgesia with epidural methadone in patients operated for hernia of the lumbar disc. Rev. Esp. Anestesiol. Reanim., v.44, p.182-185, 1997.

ISHIY, H.M.; LUNA, S.P.L.; GONÇALVES, R.C. et al. Uso da lidocaína isolada ou associada à quetamina ou ao butorfanol em anestesia epidural em cadelas submetidas à ovariosalpingohisterectomia. Rev. Bras. Cienc. Vet., v.9, p.125-127, 2002.

KAYA, F.N.; SAHIN, S.; OWEN, M.D. et al. Epidural neostigmine produces analgesia but also sedation in women after cesarean delivery. Anesthesiology, v.100, p.381-385, 2004.

KIRDEMIR, P.; OZKOCAK, I.; DEMIR, T. et al. Comparison of postoperative analgesic effects of preemptively used epidural ketamine and neostigmine. J. Clin. Anesth., v.12, p.543-548, 2000.

LAURETTI, G.R.; DE OLIVEIRA, R.; PEREZ, M.V. et al. Study of three different doses of epidural neostigmine coadministered with lidocaine for postoperative analgesia. Anesthesiology, v.90, p.1534-1538, 1999. 
LEIBETSEDER, E.N.; MOSING, M.; JONES, R.S. A comparison of extradural and intravenous methadone on intraoperative isoflurane and postoperative analgesia requirements in dogs. Vet. Anaesth. Analg., v.33, p.128-136, 2006.

MASAKI, E.; SAITO, H.; SHOJI, K. et al. Postoperative analgesic effect of epidural neostigmine and plasma cortisol and IL-6 responses. J. Clin. Anesth., v.16, p.488-492, 2004.

MARUCIO, R.; LUNA, S.P.L.; TEIXEIRA NETO, F.J. et al. Analgesic effects of epidural neostigmine with and without morphine after ovariohysterectomy in dogs. In: WORLD CONGRESS OF VETERINARY ANAESTHESIOLOGY, 9., 2006, Santos. Proceedings... Santos, 2006. p.179. (Abstract).

MASSONE, F. Anestesia local. In: MASSONE, F. (Ed). Anestesiologia veterinária: farmacologia e técnicas. 4.ed. Rio de Janeiro: Guanabara Koogan, 2003. p.33-48.

NAKAYAMA, M.; ICHINOSE, H.; NAKABAYASHI, K. et al. Analgesic effect of epidural neostigmine after abdominal hysterectomy. J. Clin. Anesth., v.13, p.86-89, 2001.

OLBRICH, V.H.; MOSING, M. A comparison of the analgesic effects of caudal epidural methadone and lidocaine in the horse. Vet. Anaesth. Analg., v.30, p.156-164, 2003.

OMAIS, M.; LAURETTI, G.R.; PACCOLA, C.A. Epidural morphine and neostigmine for postoperative analgesia after orthopedic surgery. Anesth. Analg., v.95, p.1698-1701, 2002.

OWEN, M.D.; OZSARAC, O.; SAHIN, S. et al. Low-dose clonidine and neostigmine prolong the duration of intrathecal bupivacaine-fentanyl for labor analgesia. Anesthesiology, v.92, p.361-366, 2000 .

PARRAMON, F.; GARCIA, C.H.; GAMBUS, P. et al. Postoperative patient-controlled analgesia is more effective with epidural methadone than with intravenous methadone in thoracic surgery.
Rev. Esp. Anestesiol. Reanim., v.50, p.326-331, 2003.

PRIETO-ALVAREZ, M.P.; FUENTESBELLIDO, J.G.; LOPEZ-CEBOLLADA, J. et al. Comparative study of postoperative analgesia with methadone and fentanyl in continuous peridural perfusion. Rev. Esp. Anestesiol. Reanim., v.44, p.305-309, 1997.

SHIR, Y.; ROSEN, G.; ZELDIN, A. et al. Methadone is safe for treating hospitalized patients with severe pain. Can. J. Anaesth., v.48, p.1109-1113, 2001.

TAYLOR, P. Anticholinesterase agents. In: HARDMAN, J.G.; LIMBIRD, L.E. (Eds). Goodman \& Gilman's: the pharmacological basis of therapeutics. 10.ed. New York: McGraw-Hill, 2001. p.175-191.

TORDA, T.A.; PYBUS, D.A. Comparison of four narcotic analgesics for extradural analgesia. Br. J. Anaesth., v.54, p.291-295, 1982.

TRONCY, E.; JUNOT, S.; KEROACK, S. et al. Results of preemptive epidural administration of morphine with or without bupivacaine in dogs and cats undergoing surgery: 265 cases (19971999). J. Am. Vet. Med. Assoc., v.221, p.666672, 2002.

TUNG, A.S.; YAKSH, T.L. The antinociceptive effects of epidural opiates in the cat: studies of the pharmacology and the effects of lipophilicity in spinal analgesia. Pain, v.12, p.343-356, 1982.

VALADÃO, C.A.A.; MAZZEI, S.; OLESKOVICZ, N. Injeção epidural de morfina ou cetamina em cães: avaliação do efeito analgésico pelo emprego de filamentos de von Frey. Arq. Bras. Med. Vet. Zootec., v.54, p.383389, 2002.

WAGNER, A.E. Opioids. In: GAYNOR, J.S., MUIR, WW. (Eds). Veterinary pain management. St. Louis: Mosby, 2002. p.164183.

WETMORE, L.A.; GLOWASKI, M.M. Epidural analgesia in veterinary critical care. Clin. Tech. Small Anim. Pract., v.15, p.177-188, 2000. 